\title{
Controlling the localization and migration of optical excitation
}

\author{
David L. Andrews and David S. Bradshaw \\ School of Chemistry, University of East Anglia, Norwich Research Park, Norwich NR4 7TJ, U. K.
}

\begin{abstract}
In the nanoscale structure of a wide variety of material systems, a close juxtaposition of optically responsive components can lead to the absorption of light by one species producing fluorescence that is clearly attributable to another. The effect is generally evident in systems comprising two or more light-absorbing components (molecules, chromophores or quantum dots) with well-characterised fluorescence bands at similar, differentiable wavelengths. This enables the fluorescence associated with transferred energy to be discriminated against fluorescence from an initially excited component. The fundamental mechanism at the heart of the phenomenon, molecular (resonance) energy transfer, also operates in systems where the product of optical absorption is optical frequency up-conversion.

In contrast to random media, structurally organised materials offer the possibility of pre-configured control over the delocalization of energy, through molecular energy transfer following optical excitation. The Förster mechanism that conveys energy between molecular-scale components is strongly sensitive to specific forms of correlation between the involved components, in terms of position, spectroscopic character, and orientation; one key factor is a spectroscopic gradient. Suitably designed materials offer a broad scope for the widespread exploitation of such features, in applications ranging from chemical and biological sensing to the detection of nanoscale motion or molecular conformations.

Recently, attention has turned to the prospect of actively controlling the process of energy migration, for example by changing the relative efficiencies of fluorescence and molecular energy transfer. On application of static electric fields or off-resonant laser light - just two of the possibilities - each represents a means for achieving active control with ultrafast response, in suitably configured systems. As the principles are established and the theory is developed, a range of new possibilities for technical application is emerging. For example, applications can be envisaged for new forms of all-optical switching and transistor action. There is also interest in engaging with the interplay of optical excitation and local nanoscale force, exploiting local responses to changes in dispersion forces, accompanying molecular energy transfer.
\end{abstract}

Keywords: energy transfer; nanophotonics; fluorescence; optical switching; sensors; electrodynamics; nanoscale forces; optical transistor

\section{Introduction}

When ultraviolet or visible radiation of a suitably resonant frequency shines into a non-homogeneous material containing discrete atomic, molecular or other nanoscale constituents, absorption of the light primarily populates localized electronic excited states. If there is no decay by fluorescent emission, a rapid degradation of the acquired energy typically ensues - largely a stochastic effect due to vibrational dissipation, with the released energy ultimately manifest in the form of heat. However, even in the case of fluorescent materials, the initially excited species may not directly emit radiation as they decay. In fact, a wide variety of complex materials exhibit a remarkable phenomenon: the absorption of light by one species produces fluorescence unambiguously attributable to another due to energy transfer (ET) between them. ${ }^{1,2}$ This effect has wide prevalence; it operates across a diverse and extensive range of systems - for example protein complexes, quantum dot arrays, doped crystals and solutions - and it has found numerous technical applications. It also occurs in systems where the end result of optical absorption is not the release of energy in the form of fluorescence, but optical frequency up-conversion. For the observation of such phenomena the material should contain two or more light-absorbing components (chromophores) with well-characterized absorption and fluorescence bands at broadly similar, but experimentally differentiable wavelengths. In all such materials, a localized electronic excited state will thus generally have two competing means of excited state decay - energy transfer and fluorescence. In this paper, a survey of the photophysics is presented including newly discovered control mechanisms, and a number of prospective applications are examined. 


\section{Förster transfer}

\subsection{Photophysics of energy transfer}

Electronic energy transfer is a process which effects a transportation of electronic excitation between chromophores, based on transition dipole-dipole coupling. To approach the subject in detail, we first establish the conditions for energy transfer to occur. If any neighbouring chromophore in the vicinity of a directly photo-excited, electronically activated centre has a suitably disposed electronic state, of a similar or slightly lower energy, that neighbour may acquire the major part of the initial electronic excitation through energy transfer - a process that takes place well before any further thermal degradation of the excited state energy arises. Occurrences of this mechanism are most commonly studied through spectrometric differentiation of fluorescence emerging from the initially excited energy donor and from the energy acceptor species, as illustrated in Figure 1. As will be shown in the following, the propensity for energy to be transferred in this way between any two chromophores is severely restricted by distance, and if no suitable acceptor is within reach, the donor will generally shed its energy by fluorescence or local dissipation into vibrational modes.

In energy transfer, the transitions of donor decay and acceptor excitation are generally electric-dipole allowed - other possibilities occasionally arise, but they are rare. Accordingly the theory of energy transfer, for donor-acceptor displacements beyond the region of significant wavefunction overlap, is traditionally conceived in terms of electrodynamical coupling between transition dipoles. Consider the pairwise transfer of excitation between two chromophores $D$ and $A$. In the context of this elementary mechanism, $D$ is designated the donor and $A$ the acceptor. Prior excitation of the donor generates an electronically excited species $D^{*}$, and release of the energy is accompanied by donor decay to the ground electronic state. Acquiring the energy, $A$ undergoes a transition from its ground to its excited state, $A^{*}$, which subsequently decays either in a further transfer event, or by another means such as fluorescence. Since the $D^{*}$ and $A^{*}$ excited states are real, with measurable lifetimes, the core process of energy transfer itself is fundamentally separable from the initial electronic excitation of $D$ and the eventual decay of $A$; the latter processes do not, therefore, enter into the theory of the pair transfer.

To delve more deeply into the nature of the process, it needs to be recognized that in molecular systems, the excitation of upper electronic levels usually also engage local modes of vibration, especially so when the electronic excited state has a potential energy surface displaced to any significant extent from the ground state; accordingly, in the decay process some vibrational dissipation is also usually engaged. In an inhomogeneous solid, the line-width of optical
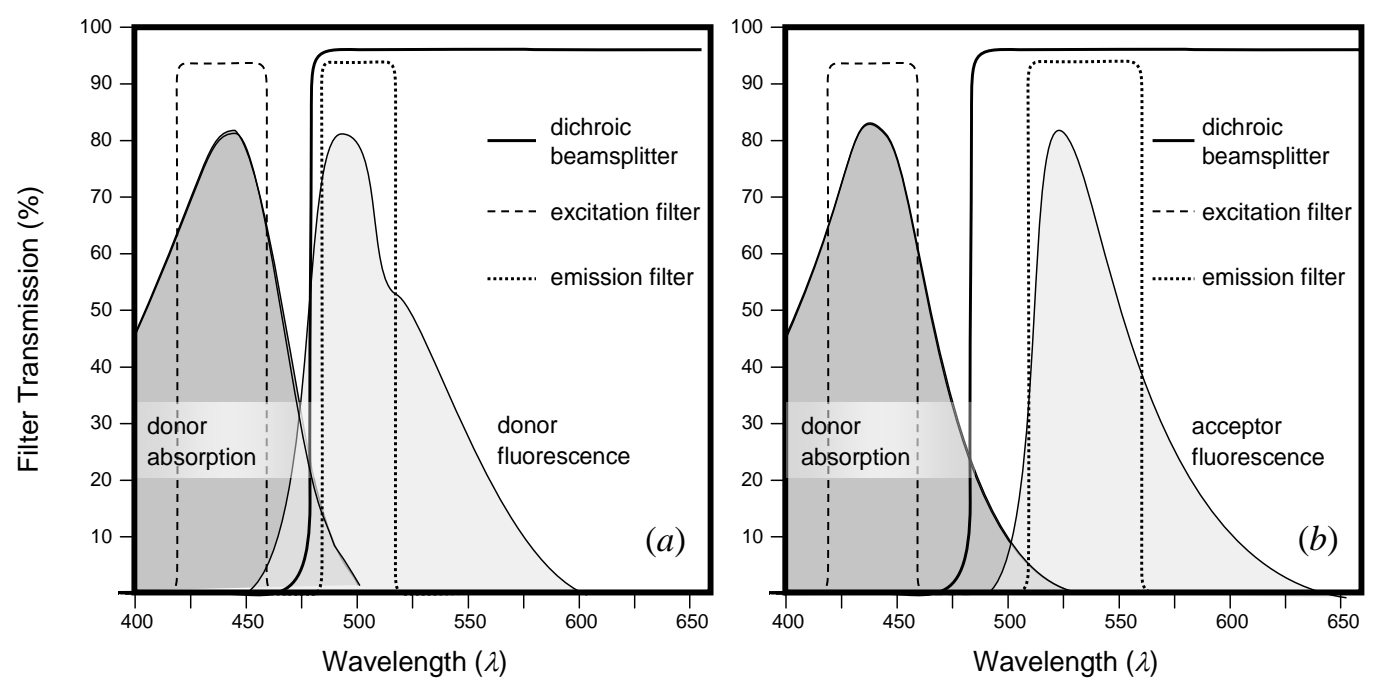

Figure 1. Typical spectral discrimination between the fluorescence from donor and acceptor species (here notionally based on a cyan fluorescent protein donor and a yellow fluorescent protein acceptor): $(a)$ the transmission characteristics of a short-wavelength filter ensure initial excitation of only the donor; a dichroic beam-splitter and another narrow emission filter ensuring that only the (Stokesshifted) fluorescence from the donor reaches a detector; $(b)$ in the same system a longer-wavelength emission filter ensures capture of only the acceptor fluorescence, following energy transfer. Adapted from ref. 2. 


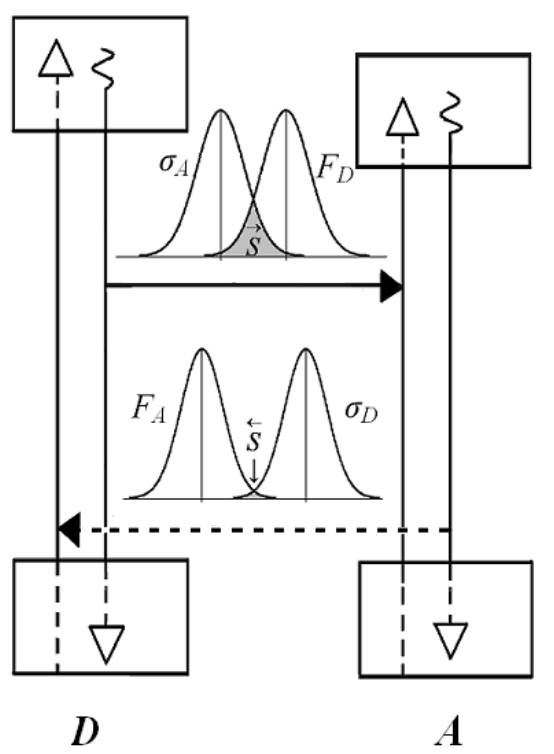

Figure 2. Energetics and spectral overlap features (top) for energy transfer from $D$ to $A$ (and below, potentially backward transfer from $A$ to $D$ ). For each chromophore $F$ denotes the fluorescence spectrum and $\sigma$ the absorption. Wavy downward lines denote vibrational dissipation. The spectral overlap for forward-directed transfer $\vec{S}$ greatly exceeds the back-transfer counterpart $\overleftarrow{S}$ Adapted from ref. 4.

transitions manifests the influence of local electronic environments; similar effects in solutions or disordered solids represent inhomogeneous interactions with a solvent or host, while the broad bands exhibited by chromophores in complex molecular systems signify extensively overlapped vibrational levels, including those associated with skeletal modes of the superstructure. In each case, energy level broadening can allow pair transfer to occur at any point within the region of overlap between the donor emission and acceptor absorption bands, as illustrated in Figure 2.

The Förster theory delivers an expression for the rate of pairwise energy transfer, $w_{\mathrm{ET}}$, valid for any donor-acceptor separation, $R$, beyond wavefunction overlap, but (as is usually the case) substantially smaller than the wavelengths of visible radiation. For systems where the common host material for the donor and acceptor has refractive index $n$, at the optical frequency corresponding to the mean transferred energy, the Förster result is as follows: ${ }^{3}$

$$
w_{\mathrm{ET}}=\frac{9 \kappa^{2} c^{4}}{8 \pi \tau_{\mathrm{FL}} n^{4} R^{6}} \int F_{D}(\omega) \sigma_{A}(\omega) \frac{\mathrm{d} \omega}{\omega^{4}} .
$$

In this expression, where $c$ is the speed of light, $F_{D}(\omega)$ denotes the normalized fluorescence spectrum of the donor, $\sigma_{A}(\omega)$ represents the linear absorption cross-section of the acceptor, and $\omega$ is an optical frequency in radians per unit time; the specific form of the integral within which they appear is known as the spectral overlap - one of the principal determinants of energy transfer efficiency. ${ }^{4}$ The second most important feature is the steep, inverse sixth power decline of rate with distance. Also featured in Eq. (1) is $\tau_{\mathrm{FL}}$, the radiative decay lifetime of the donor in the absence of transfer. This related to the measured decay lifetime $\tau$ through the fluorescence quantum yield $\eta=\tau / \tau_{\mathrm{FL}}$, where $\tau^{-1}$, expressible as $\tau^{-1}=\tau_{\mathrm{FL}}^{-1}+\tau_{\mathrm{RET}}^{-1}$, can also usefully be cast in the form;

$$
\frac{1}{\tau}=\frac{1}{\tau_{\mathrm{FL}}}+\frac{1}{\tau_{\mathrm{FL}}}\left(\frac{R_{0}}{R}\right)^{6}
$$


The last term on the right-hand side of Eq. (2) is expressed with reference to the Förster radius $R_{0}-$ the distance at which the rates of donor deactivation by energy transfer and by spontaneous fluorescence become equal. As is evident from Figure 2, the propensity for forward transfer is usually significantly greater than that for backward transfer, due to a sizeable difference in the spectral overlaps for the two processes.

The $\kappa$ factor in Eq. (1), which represents the third key rate-determining factor, depends on the orientations of the donor and acceptor with respect to each other, and with respect to their mutual displacement unit vector $\hat{\boldsymbol{R}}$. It is defined in terms of scalar products as follows:

$$
\kappa=\left(\hat{\boldsymbol{\mu}}_{D} \cdot \hat{\boldsymbol{\mu}}_{A}\right)-3\left(\hat{\boldsymbol{R}} \cdot \hat{\boldsymbol{\mu}}_{D}\right)\left(\hat{\boldsymbol{R}} \cdot \hat{\boldsymbol{\mu}}_{A}\right)
$$

For each chromophore, $\hat{\boldsymbol{\mu}}$ designates a unit vector in the direction of the appropriate transition dipole moment. The possible values of $\kappa^{2}$, as featured in Eq. (1), lie in the range $(0,4)$. In the case of fixed chromophore positions and orientations the result of Eq. (3) is evidently a function of three independent angles, shown and defined in Figure 3:

$$
\kappa=\cos \theta_{T}-3 \cos \theta_{D} \cos \theta_{A}
$$

Unfavourable orientations can thus reduce the rate of energy transfer to zero; other configurations optimize the transfer rate. It is important to note that transfer is not necessarily precluded when the transition moments lie in perpendicular directions - provided that neither is also disposed orthogonally to $\hat{\boldsymbol{R}}$.

\subsection{Applications of energy transfer}

Towards the turn of the century, energy transfer underwent a period of significant redevelopment as a technique. ${ }^{5}$ This resurgence arose mainly due to the advent of new experimentation methods, for example single-pair energy transfer, ${ }^{6}$ and further advances in instrumentation. One notable area of application is in the technology of light-harvesting materials. $^{7,8}$ Important connections with photonics have been established in pioneering work on networks of quantum dots supported in a crystal lattice, where a spectroscopic gradient assists directionality in the flow of electronic excitation ${ }^{9}-$ see Figure 4. In consequence, even when the centres are randomly distributed, such systems can lead to the production of much higher energy and more highly localised field densities, centred on the quantum dots of largest physical dimensions.

Another major use of energy transfer, based on its strong distance-dependence, exploits its capacity to supply accurate spatial information about molecular structures. This derives from a quantitative assessment of the interchromophore separations, based on comparisons between the corresponding energy transfer efficiencies. ${ }^{10-13}$ Such a technique is popularly known as a 'spectroscopic ruler'. The elucidations of molecular structure by such means usually lack information on the relative orientations of the groups involved, and as an expedient the calculations usually ignore the parameter $\kappa$ of (4). This approach appears more defensible on consideration that, even if it were to introduce a factor

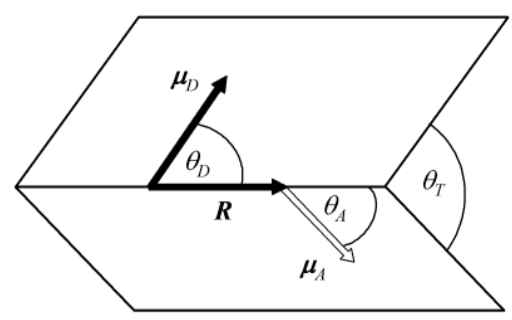

Figure 3. Relative orientations and positions of the donor and acceptor and their transition moments: Here, angles $\theta_{D}$ and $\theta_{A}$ are subtended by the donor and acceptor transition moments ( $\boldsymbol{\mu}_{D}$ and $\boldsymbol{\mu}_{A}$, respectively) against the inter-chromophore displacement vector, $\mathbf{R}$; the symbol $\theta_{T}$ is the angle between the transition moments. 


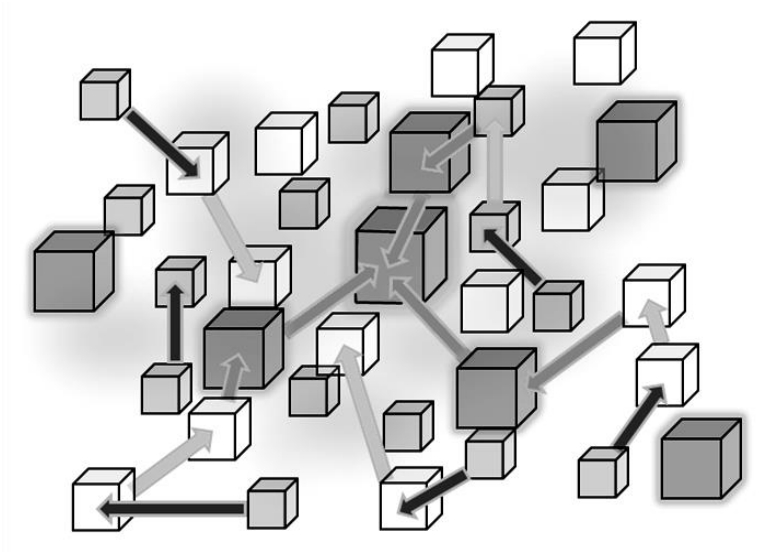

Figure 4. Scheme for an optical nanofountain comprised of $2-10 \mathrm{~nm} \mathrm{CuCl}$ quantum dots, distributed in an $\mathrm{NaCl}$ matrix. The variation in size of different quantum dots brings into effect a spectroscopic gradient directed towards the largest units. Adapted from ref. 9.

of two inaccuracy, the deduced group spacing would still be in error by only $12 \%$ (since $2^{1 / 6}=1.12$ ). Refinements to the theory to accommodate the effect of fluctuations in position or orientation of the participant groups introduce considerable complexity, although progress is being made in several areas. ${ }^{14-16}$

Studies of energy transfer variation, with changes in the proximity of one chromophore with respect to another, have become important methods for the identification of nanoscale motions in systems with suitable chromophores either intrinsic, or designedly incorporated as tags. A number of valuable energy transfer applications have arisen, notably in the sphere of molecular biology, including the detection of conformational changes and folding in proteins, ${ }^{11,17-19}$ and the inspection of intracellular protein-protein interactions. ${ }^{20,21}$ These and other such processes can be registered by selectively exciting one chromophore using laser light, monitoring either the decrease in fluorescence from that chromophore, or by a rise in the generally longer-wavelength fluorescence from the other chromophore as it adopts the role of acceptor. The judicious use of optical dichroic filters can make this energy transfer technique perfectly straightforward - see Figure 1. In cases where the two material components of interest do not have suitably overlapped absorption and fluorescence features in an optically accessible wavelength range, molecular tagging with site-specific 'extrinsic' (i.e. artificially attached) chromophores can solve the problem. Located at a molecular site of interest, and selected on the basis of a significant spectral overlap with the counterpart component, such tags can act either in the capacity of donor or acceptor. Lanthanide ions, with their characteristically prominent and sharp absorption features, prove particularly valuable in this connection. ${ }^{22}$ Again, quantum dots are also useful in this respect, particularly in view of their readily size- and composition-tunable emission across a wide spectral range. ${ }^{23,24}$

In analytical applications, the key advantage of energy transfer techniques over many others is that fluorescence measurements are highly sensitive, being made against a zero background; moreover the ultraviolet or visible signals are relatively easy to detect; they are component-specific and the required instrumentation is non-invasive. The fabrication of ET-based, analyte-specific sensors has enabled detection of a variety of species, well-established examples including dimers of functionalized calixarenes in organic solutions, ${ }^{25}$ copper(II) in aqueous solution, ${ }^{26}$ hydrogen peroxide, ${ }^{27}$ and phosgene. $^{28}$ These chemical sensors generally work on the principle of a donor-acceptor system designed such that the presence of the analyte causes the acceptor chromophore to move within closer proximity to a donor, bringing into effect an energy transfer process that would not be observed in the analyte's absence. The principle is simple: on irradiation of the system with the relevant chemical present, a strong emission from the acceptor unequivocally signals the presence of the analyte.

\subsection{Frequency up-conversion}

In a variety of structured materials the principles of electronic energy transfer extend to three-centre mechanisms, in which two distinct centres will typically act as co-donors; the acceptor gains the sum energy and then fluoresces at a shorter wavelength than the input. ${ }^{29}$ This effect has earned applications in non-parametric frequency up-conversion, often deployed as a basis for laser emission. In its necessary involvement of three chromophores rather than two, the 
process shares common ground with other photonic processes such as sensitisation, where a dopant assists the conveyance of excitation between donor and acceptor, and also quantum cutting (degenerate down-conversion), formally the time-inverse of up-conversion. Such mechanisms provide the basis for a variety of processes that have been identified in materials such as crystals, glasses and vitroceramics doped with rare earths.

\section{Active control of energy transfer}

Beyond the sphere of fluorescence and energy transfer used as tools for speciation, structure determination, molecular dynamics and optical frequency conversion, research has revealed a variety of means by which it is possible to effect experimental control over each of these fundamental photonic processes, opening up possibilities for a new realm of applications. It is well-known that a strong local field - often associated with a metallic surface - may significantly modify fluorescent decay, ${ }^{30,31}$ and some progress has been made on the theoretical engagement of a static electric field in modifying energy transfer rates. ${ }^{32}$ When the static field engages with an electronic transition, it is associated with changed selection rules - which can generally be identified with those of a two-quantum transition. The amenability of the static field-induced mechanism to controlling energy transfer is primarily based on chromophores exhibiting a (single-photon) dipole-forbidden but two-quantum allowed transition; ET does not occur to or from such a species without the presence of a static field, allowing a switchable electric field to control the delivery of energy to the acceptor.

Although there are some useful features in such static field schemes, the most attractive possibilities are undoubtedly those associated with all-optical control, where there is far greater scope for selectivity and ultrafast action. The essential elements of such schemes are illustrated in Figure 5; this section reviews some of the most recent progress.

\subsection{Optical control of fluorescence}

Passive laser control represents a catalogue of novel phenomena in which a beam of off-resonant light can engage, through a form of nonlinear optical interaction, in other, more fundamental processes. As has been discussed, the actively emitting chromophores in many materials will often have two competing means of excited state decay - energy transfer and fluorescence. Whether only one or both decay pathways are significant, the passage of a moderately intense passive laser beam may enable the optical control of the decay lifetimes, as the excited-state lifetime, $\tau$, is then expressible as follows: $\tau^{-1}=\tau_{\mathrm{FL}}^{-1}+\tau_{\mathrm{RET}}^{-1}+K I$. The first two terms on the right are as given earlier; they correspond to excited-state lifetimes due to fluorescence and spontaneous energy transfer, respectively. The newly discovered third term exhibits proportionality to $I$, the intensity of the passive beam. Our research show that the proportionality constant $K$ subsumes a passive beam dependence in both laser-controlled fluorescence and laser-assisted energy transfer; full details of the electrodynamical theory have been presented in a series of recent publications. ${ }^{33-37}$

Consider a material in which an optically excited chromophore of a specific type has no suitable acceptor to capture its energy, so that fluorescence is the principal means of excited state decay. Transmission of a passive beam through the

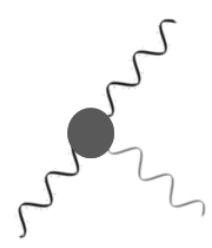

(a)

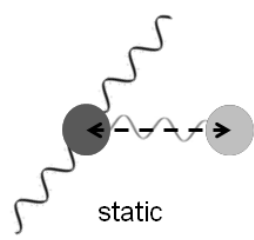

(b)

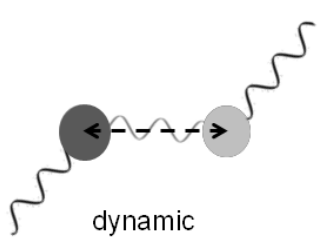

(c)

Figure 5. (a) Optically controlled fluorescence is brought into effect by engaging the emission process with forward scattering of photons in the passive laser beam. Optically controlled energy transfer is mediated by two sub-mechanisms, static and dynamic; $(b)$ in the static mechanism the transfer of energy from donor to acceptor engages with forward scattering of the passive beam by the donor, as shown, or by the acceptor; $(c)$ in the case of dynamic coupling the transfer of energy engages with forward scattering through both donor and acceptor. 
system, during the excited state lifetime, can characteristically modify the intensity of fluorescent emission, as well as its associated optical polarization and temporal behaviour. The mechanistic conditions are entirely different from those that would operate under the delivery of resonant radiation, which would promote stimulated emission. Here, no net absorption or stimulated emission occurs since the throughput beam is off-resonance and therefore emerges unchanged. Specifically, elastic forward-scattering of the off-resonant light couples with the fluorescence emission; the probe laser confers optical nonlinearity on the emission process. According to our calculations, fluorescence lifetimes will typically be reduced by about 20 percent for an input laser irradiance of $2 \times 10^{11} \mathrm{~W} \mathrm{~cm}^{-2}$, and proportionately more for higher intensities; a maximum is imposed by the pumping rate of the laser that initially excites the system. Even at $10^{10} \mathrm{~W} \mathrm{~cm}^{-2}$, such changes in fluorescence lifetime are readily measurable with modulation detection techniques. One of the most commonly measured structural parameters, the degree of fluorescence anisotropy, which measures the difference in fluorescence intensities through polarisers parallel and perpendicular to the polarization of an excitation beam, also undergoes a characteristic change due to the interaction with the passive beam.

We can envisage optically controlled fluorescence as an analytical tool. Using a tunable laser source, wavelength variation of the passive radiation will offer an additional dimension to sample interrogation, helping to differentiate samples that appear indistinguishable by conventional optical methods. In addition, such non-intrusive methods might be used to improve spatial resolution in fluorescence imaging applications, allowing the characterization of high-grade materials such as microcrystalline polymorphs.

\subsection{Optical transistors}

There is a particularly attractive prospect associated with laser-controlled fluorescence. Our research has recently shown that the output of a three-level laser, optically pumped just below threshold, may be strongly enhanced on an ultrafast timescale through laser-controlled fluorescence. ${ }^{38}$ Analysis demonstrates that pulses of light from a passive laser, acting as an off-resonant input, will modify the amplification kinetics of the active medium and can thereby trigger laser action. The results of calculations for a representative three-level system highlight a significant potential for all-optical transistor action. For a constant pumping rate, at a level indicated by the dotted vertical line (see Figure 6), the system operates below threshold when there is no input laser beam. Introducing a passive beam with an irradiance approaching $2 \times 10^{11}$ $\mathrm{W} \mathrm{cm} \mathrm{c}^{-2}$, the output climbs by approximately 14 orders of magnitude (rising to 16 orders if the input intensity is doubled) - a rise that is absolutely typical of a laser operating around threshold. Transistor action with respect to the passive beam is clearly exhibited. The beauty of this scheme is that it is not limited in principle to an operation with any specific material; the challenge is now to find three-level systems optimizing the necessary nonlinear optical characteristics.

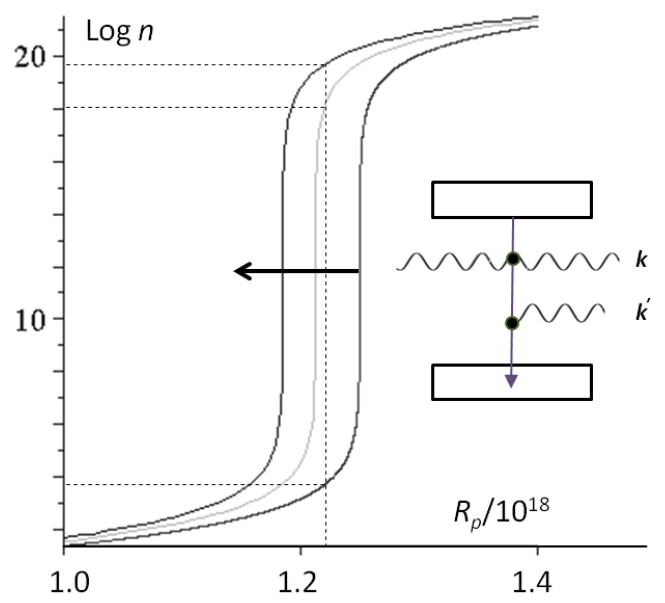

Figure 6. $\log n$, where $n$ is the number of cavity photons, plotted against pumping rate, $R_{p}$, in the absence (right-hand plot) and presence of a passive beam, the latter with irradiance $2 \times 10^{11} \mathrm{~W} \mathrm{~cm}^{-2}$ (middle plot) and $4 \times 10^{11} \mathrm{~W} \mathrm{~cm}^{-2}$ (left-hand plot). The lasing threshold reduces with increasing intensity of the passive beam. With suitable pumping, the passive beam produces a shift to abovethreshold operation (upper dotted lines). Inset: Corresponding energy level scheme: the $k$ ' and $k$ waves denote the emission and passive input, respectively; electronic states are signified by rectangular boxes. 


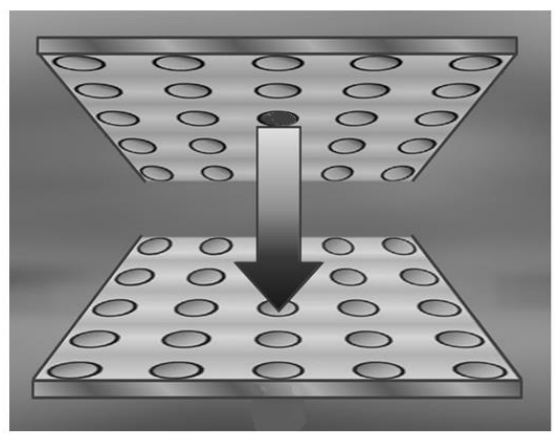

(a)

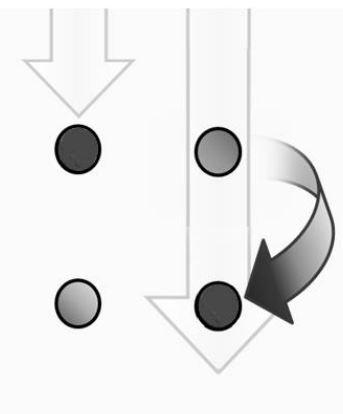

(c)

Figure 7. Depiction of a two-dimensional square-lattice configuration for an all-optical switching device: $(a)$ side-view (not showing spacer layer between the two arrays); the initial excitation (dark grey dot) is transferred only to its partner directly underneath: $(b)$ with

the passive beam yet to enter there is no transfer of excitation, $(c)$ with the beam passing though the system transfer does occur.

\subsection{Optical control of energy transfer}

In the case of energy transfer, a passive laser beam may enhance (or diminish) the process through a conferred optical response known as laser-assisted resonance energy transfer. This involves elastic forward-scattering of the passive beam, again without any net absorption or stimulated emission, and again the efficiency of this process scales linearly with laser intensity. Having the ability to exert optical control over the migration of energy provides a basis for all-optical switching in an adapted form of laser-assisted energy transfer, where the spontaneous process is completely disabled. This can be achieved in an arrangement where the vector displacement of the acceptor from the donor - and each of their transition dipoles - are all mutually perpendicular; spontaneous energy transfer is then excluded by geometry (Figure 7). Alternatively, spontaneous transfer will be inhibited when the electronic transition in either the donor or acceptor is one-photon forbidden but two-photon allowed. In either case, the switching action induced by the transport of energy is activated only when the passive beam is "on".

Modelling has indicated that an efficient, all-optical heterostructure device built on a two-dimensional square-lattice configuration can operate with readily achievable laser irradiances in the range $10^{10}-10^{12} \mathrm{~W} \mathrm{~cm}^{-2}$. The parallelprocessing capability of such a device introduces a variety of possible applications. For example, pixel-based images, written by donor excitation, might be controllably transferred with high fidelity to an acceptor film. To build such a switching device, researchers could exploit recent advances in the construction of quantum dot arrays, with juxtaposed arrays sandwiching an optically transparent spacer layer. Technical realizations will determine the most suitable nanofabrication method, such as dip-pen nanolithography. In the realm of optical communications, further possibilities arise for the system to act as an ultrafast information buffer; "slow-light" methods have also been considered for similar aims, but with limited success. Optically controlled ET has the positive characteristics of viable operation at short visible/ultraviolet wavelengths without expensive, non-standard optical elements; above all, it offers a high-information density and ultrafast response with high repetition rate and high efficiency.

\subsection{Energy transfer-driven dispersion interactions}

Surprisingly little attention seems to have been given to the fact that each act of energy transfer produces secondary changes in the local electronic environment of the donor and acceptor, shifting the electromagnetic interactions between participant chromophores and producing modified intermolecular forces. From recent theory it emerges that energy transfer, especially when it occurs between chromophores with electronically dissimilar properties (such as differences in polarizability), can generate significant changes in the intermolecular potentials. ${ }^{39}$

Since the form of the dispersion interaction depends on the electronic states of the molecular participants, the dispersion force between neutral molecules is clearly subject to change during the course of absorption and energy transfer. The local electronic environments will first experience change upon optical excitation of any donor, the associated modification of electromagnetic interactions producing modified intermolecular forces. In general, a degree of local movement can be expected as the system accommodates to a modified potential energy surface. If the absorbed 


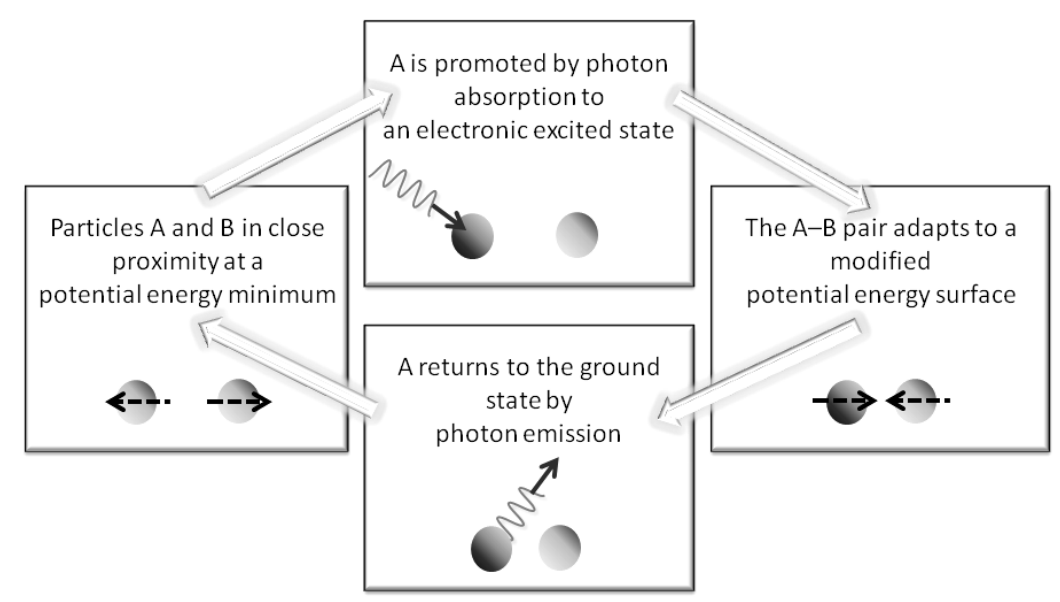

Figure 8. Simplest case of dispersion-driven response to optical excitation, without incurring energy transfer.

energy then transfers to a neighbouring acceptor unit of another species, the local electronic environment will suffer further change, and a further compensating motion can be expected to occur. In particular, in a solid-state environment where intermolecular forces are balanced in an equilibrium configuration, any changes associated with the migration of local electronic excitation should effectively act as a small perturbation to the equilibrium of intrinsic forces, producing measurable displacements - see Figure 8 as an example.

\section{Discussion}

It is remarkable that widely varying control phenomena owe their origin to a fundamentally similar form of electromagnetic interaction, namely the stimulated re-emission of passive, off-resonant transmitted light - indeed, interactions of a similar form are known to be responsible for the phenomenon known as "optical binding". Amongst the numerous, very different applications that are beginning to emerge we anticipate that the work on optical transistor action, based on the passive control of fluorescence, may break new ground for the broader development of all-optical methods to control photon absorption and emission. Such opportunities are not limited to device applications; since operation does not require atomic motion, and switching times are comparable to an excited state decay, there are clear advantages over the current generation of molecular switches - devices based on laser-activated emission will be much faster in operation and cycle time. In the ongoing progress of optoelectronics, a new generation of molecular devices will emerge, tailored for use in optical communications and IT. Finally, there is plenty of scope to explore potential sensor applications of the novel nano-optomechanical effects associated with energy transfer. It seems not too much of a exaggeration to consider that a whole new field of photonic applications is on the horizon.

\section{Acknowledgment}

The authors would like to thank the Leverhulme Trust for funding this research.

\section{References}

[1] Scholes, G. D., "Long-range resonance energy transfer in molecular systems,” Annu. Rev. Phys. Chem. 54, 57-87 (2003).

[2] Andrews, D. L. and Bradshaw, D. S., "Resonance energy transfer," in [Encyclopedia of Applied Spectroscopy], Andrews, D. L., Ed., Wiley-VCH, Weinheim, 533-554 (2009). 
[3] Demidov, A. A. and Andrews, D. L., in [Encyclopedia of Chemical Physics and Physical Chemistry], Moore, J. H. and Spencer, N. D., Eds, Institute of Physics, Bristol, vol. 3, 2701-2715 (2001).

[4] Andrews, D. L. and Rodríguez, J., "Resonance energy transfer: Spectral overlap, efficiency and direction,” J. Chem. Phys. 127, 084509 (2007).

[5] Selvin, P. R., “The renaissance of fluorescence resonance energy transfer,” Nat. Struct. Bio. 7, 730-734 (2000).

[6] Ha, T., Enderle, T., Ogletree, D. F., Chemla, D. S., Selvin, P. R. and Weiss, S., "Probing the interaction between two single molecules: Fluorescence resonance energy transfer between a single donor and a single acceptor," Proc. Natl. Acad. Sci. USA 93, 6264-6268 (1996).

[7] Olaya-Castro, A. and Scholes, G. D., "Energy transfer from Forster-Dexter theory to quantum coherent lightharvesting," Int. Rev. Phys. Chem. 30, 49-77 (2011).

[8] Bradshaw, D. S. and Andrews, D. L., "Mechanisms of light energy harvesting in dendrimers and hyperbranched polymers," Polymers 3, 2053-2077 (2011).

[9] Kawazoe, T., Kobayashi, K. and Ohtsu, M., “Optical nanofountain: A biomimetic device that concentrates optical energy in a nanometric area,” Appl. Phys. Lett. 86, 103102 (2005).

[10] Hohng, S., Joo, C. and Ha, T., “Single-molecule three-color FRET,” Biophys. J. 87, 1328-1337 (2004).

[11] Schuler, B., "Single-molecule fluorescence spectroscopy of protein folding," ChemPhysChem 6, 1206-1220 (2005).

[12] Koushik, S. V., Chen, H., Thaler, C., Puhl III, H. L. and Vogel, S. S., "Cerulean, venus and venusY67C FRET reference standards,” Biophys. J. 91, L99-L101 (2006).

[13] Zhang J. and Allen, M. D., "FRET-based biosensors for protein kinases: Illuminating the kinome," Mol. Biosyst. 3, 759-765 (2007).

[14] Yu, Z. G., "Fluorescent resonant energy transfer: Correlated fluctuations of donor and acceptor," J. Chem. Phys. 127, 221101 (2007).

[15] Isaksson, M., Norlin, N., Westlund, P.-O. and Johansson, L. B.-Å., "On the quantitative molecular analysis of electronic energy transfer within donor-acceptor pairs,” Phys. Chem. Chem. Phys. 9, 1941-1951 (2007).

[16] Jang, S., "Generalization of the Förster resonance energy transfer theory for quantum mechanical modulation of the donor-acceptor coupling," J. Chem. Phys. 127, 174710 (2007).

[17] Talaga, D. S., Lau, W. L., Roder, H., Tang, J., Jia, Y. W., DeGrado, W. F. and Hochstrasser, R. M., “Dynamics and folding of single two-stranded coiled-coil peptides studied by fluorescent energy transfer confocal microscopy,” Proc. Natl. Acad. Sci. USA 97, 13021-13026 (2000).

[18] Heyduk, T., "Measuring protein conformational changes by FRET/LRET," Curr. Opin. Biotechnol. 13, 292-296 (2002).

[19] Schuler, B. and Eaton, W. A., "Protein folding studied by single-molecule FRET," Curr. Opin. Struct. Biol. 18, 16-26 (2008).

[20] Parsons, M., Vojnovic, B. and Ameer-Beg, S., "Imaging protein-protein interactions in cell motility using fluorescence resonance energy transfer (FRET),” Biochem. Soc. Trans. 32, 431-433 (2004).

[21] You, X., Nguyen, A. W., Jabaiah, A., Sheff, M. A., Thorn, K. S. and Daugherty, P. S., "Intracellular protein interaction mapping with FRET hybrids," Proc. Natl. Acad. Sci. USA 103, 18458-18463 (2006). 
[22] Selvin, P. R., "Principles and biophysical applications of lanthanide-based probes," Annu. Rev. Biophys. Biomol. Struc. 31, 275-302 (2002).

[23] Chan, W. C. W., Maxwell, D. J., Gao, X., Bailey, R. E., Han M. and Nie, S., "Luminescent quantum dots for multiplexed biological detection and imaging," Curr. Opin. Biotechnol. 13, 40-46 (2002).

[24] Clapp, A. R., Medintz, I. L. and Mattoussi, H., "Förster resonance energy transfer investigations using quantumdot fluorophores," Chem. Phys. Chem. 7, 47-57 (2006).

[25] Castellano, R. K., Craig, S. L., Nuckolls, C. and Rebek, J., "Detection and mechanistic studies of multicomponent assembly by fluorescence resonance energy transfer,” J. Am. Chem. Soc. 122, 7876-7882 (2000).

[26] Cano-Raya, C., Fernández-Ramos, M. D. and Capitán-Vallvey, L. F., "Fluorescence resonance energy transfer disposable sensor for copper(II)," Anal. Chim. Acta 555, 299-307 (2006).

[27] Albers, A. E., Okreglak, V. S. and Chang, C. J., "A FRET-based approach to ratiometric fluorescence detection of hydrogen peroxide," J. Am. Chem. Soc. 128, 9640-9641 (2006).

[28] Zhang, H. and Rudkevich, D. M., "A FRET approach to phosgene detection," Chem. Commun., 1238-1239 (2007).

[29] Andrews, D. L. and Jenkins, R. D., "A quantum electrodynamical theory of three-center energy transfer for upconversion and downconversion in rare earth doped materials," J. Chem. Phys. 114, 1089-1100 (2001).

[30] Anger, P., Bharadwaj, P. and Novotny, L., "Enhancement and quenching of single-molecule," Phys. Rev. Lett. 96, 113002 (2006).

[31] Zhao, Y., Jiang, Y. and Fang, Y., "Quenching and enhancement of fluorescence of fullerene molecules on gold particle," Chem. Phys. 323, 169-172 (2006).

[32] Andrews, D. L. and Bradshaw, D. S., "Theory of directed transportation of electronic excitation between single molecules through photonic coupling," Proc. SPIE 6999, 69990C (2008).

[33] Bradshaw, D. S. and Andrews, D. L., "Optically controlled resonance energy transfer: Mechanism and configuration for all-optical switching," J. Chem. Phys. 128, 144506 (2008).

[34] Bradshaw, D. S. and Andrews, D. L., "All-optical switching based on controlled energy transfer between nanoparticles in film arrays,” J. Nanophoton. 3, 031503 (2009).

[35] Bradshaw, D. S. and Andrews, D. L., "Mechanism for optical enhancement and suppression of fluorescence," J. Phys. Chem. A 113, 6537-6539 (2009).

[36] Bradshaw, D. S. and Andrews, D. L., "All-optical switching between quantum dot nanoarrays," Superlatt. Microstruct. 47, 308-313 (2010).

[37] Bradshaw, D. S. and Andrews, D. L., “All-optical control of molecular fluorescence," Phys. Rev. A 81, 013424 (2010).

[38] Andrews, D. L. and Bradshaw, D. S., "Off-resonant activation of optical emission," Opt. Commun. 283, 43654367 (2010).

[39] Andrews, D. L., Bradshaw, D. S., Leeder, J. M. and Rodríguez, J., "Dynamics of the dispersion interaction in an energy transfer system,” Phys. Chem. Chem. Phys. 10, 5250-5255 (2008). 